\section{Rudiments of physics}

Elementary Particles and Symmetries. (Documents on Modern Physics.) By Lewis Ryder. Pp. xxvi +249. (Gordon and Breach : New York, London and Paris, December 1975.) $£ 9.80 ; £ 5.00$ (International Student Edition).

LEWIS RYDER's book grew out of lectures given to third-year undergraduates at the University of Kent, and it shows. In my opinion, it is not enough simply to tell students about particle physics. I share what I take to be the author's view that it is preferable, or at least equally important, to teach them how to calculate something - almost anything will do! Any like-minded teacher will find a wealth of material in this admirable book. Of course the topic of symmetries is well suited to this task. The discrete symmetries (time reversal, charge conjugation, parity reversal), the additive conservation laws (charge, strangeness) and the non-abelian groups (isospin, $\mathrm{SU}_{3}$ ) are all covered and applied to concrete examples of the strong, weak and electromagnetic interactions. Ten of the thirteen chapters are essentially free of phrases

\section{Organic mechanics}

Mechanical Design in Organisms. By S. A. Wainwright, W. D. Biggs, J. D. Currey and J. M. Gosline. Pp. xii +425 . (Arnold: London, January 1976.) $£ 8.50$.

THIS is an ambitious work which brings together much biological information about structural macromolecules and skeletal tissues with the increasing knowledge of their mechanics. It is a book for the specialist and general reader alike, although comparatively few biologists will be sufficiently familiar with materials science, or adequately equipped mathematically, to follow all the engineering phenomena. The first part deals with various tensile, pliant and rigid materials incorporated into skeletal systems. The second part looks into the design of skeletal elements and mechanical support systems, and the third part examines organism-environment interactions. The literature has been assiduously searched and digested, but the book is not an uncritical collection of research results. The authors have rigorously argued their central theme: that structural elements are designed to carry out specific functions in an environment of stresses and strains. Biologists have long appreciated this interplay between form and function, but for many the concept is possibly more an article of faith than a com- like: "It can be shown that...", which usually herald the onset of the narrative approach. And en route quite a lot of basic information, including some modern developments, is imparted. One surprising omission occurs in the section on the deep inelastic electron-proton scattering data. Having explained why these very important experiments suggest that the nucleon "is like a pomegranate, not like a jelly", I expected, but did not find, some treatment of the naive quark-parton model. Showing how the data suggests that the "seeds" of the pomegranate have onethird integral charges, would have lent further weight to the subsequently raised hot question of particle physics: do quarks actually exist? Towards the end the narrative style inevitably prevails. But even in the last chapter, on the unified theories of weak and electromagnetic interactions, there is enough to show a (keen) beginner how to embark on his own rudimentary model building.

All in all, I think that the book is remarkably successful, and that it caters well for its intended market. The price too is reasonable by current standards, at least for the paperback edition.

D. Bailin

plete understanding. This book provides the comprehension as well as the creeds.

Editorial and technical production reaches a high standard. The book is written throughout in a simple, lucid style (with the occasional touch of humour) making it easy and enjoyable to read. It is elegantly illustrated with numerous drawings and diagrams combining pleasing clarity with meticulous draughtsmanship. I detected few errors, but a curious recurrent mistake is the term 'keratin sulphate' which should be keratan sulphate, or keratosulphate. Naturally there are some points of contention. The dogmatic statement (p105) that $\alpha$ chitin alone exists in Arthropoda is some three years out of date: well-defined examples of $\beta$ and $\gamma$ chitin are present in some insect peritrophic membranes and larval cocoons. The ' $9+2$ ' theory for the structure of $\alpha$-keratin microfibrils is given another airing, but there is no mention of the 'ring-core' model which fits more closely the $\mathrm{X}$-ray diffraction data and computational studies.

Mechanical Design in Organisms is bound to attract and stimulate considerable interest among biologists and others. It will be an important reference hook for some time to come, and as a text it will provide a basis for interdisciplinary teaching of new topics in biophysics and biology degree courses.

W. Kenchington

\section{Molecular spectroscopy}

Theory of Molecular Spectroscopy. Vol. 1: Quantum Mechanics and Group Theory of Vibrating and Rotating Molecules. By C. J. H. Schutte. Pp. xvi+512. (North-Holland: Amsterdam and Oxford; American Elsevier: New York, 1976.) Dfl. 170; $\$ 67.95$.

THIS is volume 1 of a two volume work and it is not too easy to judge in isolation. It is a strongly mathematical introduction covering vector spaces, matrices, group theory, Hamiltonians in general, both the classical and quantum mechanical form of vibration-rotation interactions, diatomic potential functions and their evaluation, and the interaction of radiation with matter.

The mathematical treatment is thorough and a number of advanced topics are covered, such as the Wilson and Howard Hamiltonian and its modification by Watson, and also the Rydberg-Klein-Rees method of fitting potential curves. The treatment is mostly sound and comparatively free of misprints, and the first impression, strengthened by the early chapter on group theory, is favourable. But $I$ became increasingly unhappy as I delved deeper. It is doubtful whether a standard treatment of the hydrogen atom would be expected in an expensive book on rotating molecules. And as the text gets to real problems, rather than abstract algebra, a lack of authority becomes apparent. Section 1.4 on normal co-ordinates is especially confused; for example, Schutte's use of Cartesians weighted with the square roots of the masses means that the kinetic energy matrix should be the unit matrix, but this is not recognised in this text. And the statement (p241) that the energy "is then $k R^{2}$, since $k$ is defined as $\mathrm{Nm}^{-1}$ " leads to a lack of confidence as does that on p307: "If a molecule does not have a permanent dipole moment, the selection rules for dipole radiation prohibits the occurrence of an infrared spectrum".

There is a need for a comprehensive advanced treatise on the theory of ro-vibration, but part of the difficulty of writing is to choose the important features and give them once only in a modern and consistent form. This text, which has some good sections, tends to give a rather indigestible slab of everything. The price is high and the second companion volume, which will contain the more specific features, is not likely to be less.

D. H. Whiffen 